\title{
Ulinastatin protects against lipopolysaccharide-induced cardiac microvascular endothelial cell dysfunction via downregulation of IncRNA MALAT1 and EZH2 in sepsis
}

\author{
ZHAOXIA YU $^{1}$, AISA RAYILE ${ }^{2}$, XIANGYANG ZHANG ${ }^{2}$, YING LI $^{1}$ and QIANG ZHAO $^{2}$ \\ ${ }^{1}$ Intensive Care Unit, ${ }^{2}$ Department of Cardiac Center, The First Affiliated Hospital of \\ Xinjiang Medical University, Urumqi, Xinjiang 830054, P.R. China
}

Received September 26, 2016; Accepted March 3, 2017

DOI: $10.3892 /$ ijmm.2017.2920

\begin{abstract}
The present study aimed to evaluate the effects of ulinastatin on the permeability and apoptosis of lipopolysaccharide (LPS)-induced cardiac microvascular endothelial cells (CMVECs), and investigate its molecular mechanisms in sepsis. The sepsis rat model was induced by cecal ligation and puncture (CLP), and rat CMVECs were isolated and treated with LPS or/and ulinastatin. Then, cell permeability was evaluated by transendothelial electrical resistance, reactive oxygen species (ROS) levels were assessed by 2,7-dichlorofluorescein diacetate, cell apoptosis was detected using Annexin V-FITC apoptosis detection kit, and the expression levels of Bcl-2, Bax, caspase-3, metastasis-associated lung adenocarcinoma transcript 1 (MALAT1) and EZH2 were detected by RT-qPCR and/or western blotting. In addition, the relationship of MALAT1 and EZH2 was evaluated by RNA immunoprecipitation (RIP) assay and chromatin immunoprecipitation (ChIP) assay. Compared with LPS-induced CMVECs, treatment with ulinastatin significantly reduced CMVEC permeability and the percentage of apoptotic cells, as well as an increased level of Bcl-2 and inhibited the levels of ROS, caspase- 3 and Bax (all $\mathrm{p}<0.05$ ). In addition, long non-coding RNA (lncRNA) MALAT1 was confirmed to interact with EZH2 in CMVECs. Overexpression of 1ncRNA MALAT1 and EZH2 was found in the hearts of the sepsis rat and LPS-induced CMVECs, while ulinastatin inhibited the upregulation of IncRNA MALAT1 and EZH2 in the LPS-induced CMVECs (all $\mathrm{p}<0.05$ ). Ulinastatin protected against LPS-induced CMVEC cell hyperpermeability and apoptosis via downregulation of lncRNA MALAT1 and EZH2 in sepsis.
\end{abstract}

Correspondence to: Professor Xiangyang Zhang, Department of Cardiac Center, The First Affiliated Hospital of Xinjiang Medical University, 137 South Liyushan Road, Urumqi, Xinjiang 830054, P.R. China

E-mail: xiangyangzhang13@sina.com

Key words: sepsis, cardiac microvascular endothelial cells dysfunction, long non-coding RNA metastasis-associated lung adenocarcinoma transcript 1 , ulinastatin

\section{Introduction}

Sepsis is a severe inflammatory disease in response to bacterial infection (1). It is characterized by systemic inflammatory response syndrome (SIRS) and is frequently caused by hemorrhage, trauma and abdominal surgery (1). In the intensive care unit, severe sepsis is considered as a leading cause of death due to the occurrence of SIRS and multiple organ dysfunctions $(2,3)$. Thus, it is imperative to understand the underlying pathogenesis and search for effective intervention and therapy for sepsis.

Previous study has demonstrated that cardiac dysfunction is induced in $~ 50 \%$ of critically ill patients with sepsis, and the recovery of cardiac function is considered as a key predictor for survival (4). It is reported that cardiac microvascular dysfunction is associated with cardiac dysfunction in patients with sepsis (5). Importantly, cardiac microvascular endothelial cells (CMVECs), accounting for $1 / 3$ of all heart cells, exert an important influence on the normal condition of coronary microvessels and adjacent cardiomyocytes (6). In addition, CMVEC dysfunction, such as hyperpermeability and apoptosis caused by inflammatory responses, is found in myocardial ischemia/reperfusion injury $(7,8)$. Although cardiac microvascular dysfunction is confirmed in sepsis, few studies have investigated the function of CMVECs in sepsis.

Ulinastatin, a protease inhibitor, exhibits anti-inflammatory activity by inhibiting the expression of pro-inflammatory factors and blocking the signaling pathways related to inflammation (9). Several animal experiments have demonstrated a protective role of ulinastatin against sepsis by suppressing the inflammatory response (10-12). Randomized controlled clinical studies have further revealed that ulinastatin can significantly improve organ failure and reduce mortality in patients with sepsis $(13,14)$. However, it is still unclear whether the role of ulinastatin in sepsis is associated with the function of CMVECs.

Currently, increasing evidence has demonstrated that long non-coding RNAs (IncRNAs) are involved in various diseases (15), including neurodegenerative diseases (16), cardiovascular diseases (17) and cancers (18) by regulating target gene transcription. Understanding the underlying mechanisms of lncRNAs in sepsis may contribute to an effective 
treatment for sepsis. Metastasis-associated lung adenocarcinoma transcript 1 (MALAT1) was originally known as an lncRNA in patients with non-small cell lung cancer (NSCLC) who have a high risk of metastasis. A recent study reported that MALAT1 is associated with cell proliferation and migration in cancer cells (19). Nevertheless, its role in CMVEC dysfunction of sepsis is still poorly investigated. In the present study, a sepsis model in the rat was induced by cecal ligation and puncture (CLP), and rat CMVECs were isolated and treated by lipopolysaccharide (LPS) or/and ulinastatin. We aimed to evaluate the effects of ulinastatin on the permeability and apoptosis of LPS-induced CMVECs and investigate its molecular mechanisms.

\section{Materials and methods}

Ethics statement. Healthy male Sprague-Dawley (SD) rats ( 100 g of body weight) were purchased from Beijing Vital River Laboratory Animal Technology Co., Ltd. (Beijing, China). Approval from the Institutional Animal Care and Use Committee of the First Affiliated Hospital of Xinjiang Medical University was obtained prior to all animal experiments.

Animal model of sepsis. A total of 50 SD rats were randomly assigned into two groups: a sham group $(n=10)$ and a sepsis group $(n=40)$. All rats were acclimatized for 3-5 days before the experiments. The rat sepsis model was established using CLP according to a previously described (20). CLP induced intra-abdominal infection and the release of LPS, resulting in SIRS and sepsis. Briefly, the rats were anesthetized by an intraperitoneal injection of $10 \%$ chloral hydrate $(3 \mathrm{ml} / \mathrm{kg}$; Sigma, St. Louis, MO, USA) and fixed in a supine position. A $1.5-\mathrm{cm}$ longitudinal incision was made along the abdominal midline, then the caecum was exposed and separated. For rats in the sepsis group, the caecum below the ileocaecal valve was ligated using 3.0 suture. Then, a 18-gauge needle was used to puncture through the caecum 3 times, and the feces was extruded by gently squeezing the caecum. Lastly, the separated caecum was returned to the abdominal cavity and the incision was sutured layer by layer. Rats in the sham group were treated with similar procedures but without ligation or puncture. The entire operation was carried out under aseptic conditions. The rats were sacrificed $24 \mathrm{~h}$ post-operatively, and the heart tissues were collected for the following experiments.

Isolation of CMVECs. CMVECs were isolated from SD rats following a previously reported method (21). In brief, two SD rats were anesthetized and then intraperitoneally injected with heparin (300 IU/g; Sigma) for $20 \mathrm{~min}$. Then, the rats were sacrificed by cervical dislocation. After sterilization with $75 \%$ alcohol, the thoracic cavity was opened. Then, the heart was removed and rinsed with sterile D-Hanks' containing heparin. Next, the connective tissue, atria, right ventricle and interventricular septum were removed, and epicardial and endocardial cells from the left ventricle were devitalized by immersing in $75 \%$ ethanol for $20-30 \mathrm{sec}$. The remaining tissue was minced and rinsed with D-Hanks', and then incubated in $0.02 \%$ collagenase type II (dissolved in D-Hanks') for $30 \mathrm{~min}$ and $0.02 \%$ trypsin (both from Sigma) for another $10 \mathrm{~min}$ at $37^{\circ} \mathrm{C}$. The dissociated cells were centrifuged at $1,000 \mathrm{rpm}$ for $10 \mathrm{~min}$, and then resuspended in Dulbecco's modified Eagle's medium (DMEM; Invitrogen, Cambridge, MA, USA) containing $15 \%$ fetal bovine serum (FBS). Lastly, the cells were cultured in laminin-coated dishes (Corning, Inc., Corning, NY, USA).

Cell treatment. LPS was used to induce the sepsis cell model. To determine the optimum inducement time and concentration, the primary CMVECs were treated with various concentrations $(0,10,50,100 \mathrm{ng} / \mathrm{ml}, 1$ and $2 \mu \mathrm{g} / \mathrm{ml})$ for $0,3,6,9$ and $12 \mathrm{~h}$, respectively. To investigate the effect of ulinastatin on the LPS-induced CMVECs, the CMVECs were treated with LPS and LPS + ulinastatin $(1000,3000$ or $5000 \mathrm{U} / 1)$, respectively. In addition, the treated cells were also transfected with the lncRNA MALAT1 overexpression vector (named as pc-MALAT1; RiboBio, Guangzhou, China) using Lipofectamine 2000 (Invitrogen).

Assessment of cell permeability. To evaluate cell permeability, transendothelial electrical resistance (TER) was measured using STX2 electrode and EVOM2 meter (World Precision Instruments, Sarasota, FL, USA). CMVECs cells were seeded on fibronectin-coated Transwell membrane inserts $(0.4-\mu \mathrm{m}$ pore size; Corning, Inc.). Then, confluent CMVECs were treated with LPS, LPS + ulinastatin or LPS + ulinastatin + lncRNA MALAT1 overexpression vector, respectively. The value of TER was calculated as the resistance value multiplied by the membrane area.

Cell apoptosis assay. The Annexin V-FITC apoptosis detection kit (Invitrogen) was used to quantify cell apoptosis. Briefly, the treated cells were collected by digestion with Trypsin and centrifugation at 1,500 rpm for $6 \mathrm{~min}$. After being washed with phosphate-buffered saline (PBS), the cells were mixed with FITC-Annexin $\mathrm{V}$ and propidium iodide (PI) for $15 \mathrm{~min}$ at $25^{\circ} \mathrm{C}$ in the dark. Cells were then added to $400 \mu 11 \mathrm{X}$ binding buffer and detected using a flow cytometer. Annexin V-positive and PI-negative cells were considered as apoptotic cells.

Reactive oxygen species (ROS) assay. The ROS level was assessed by 2,7-dichlorofluorescein diacetate (DCFH-DA; Sigma). After being washed with PBS, the treated cells were incubated with serum-free DMEM containing $10 \mu \mathrm{M}$ DCFH-DA for $20 \mathrm{~min}$ at $37^{\circ} \mathrm{C}$ in the dark. Next, the cells were collected and analyzed using a Spectra Max M5 microplate reader (Molecular Devices, Sunnyvale, CA, USA). The fluorescent intensities were measured at a $535 \mathrm{~nm}$ absorbance.

Real-time-quantitative polymerase chain reaction ( $R T$ - $q P C R)$ analysis. Total RNA extraction was carried out using TRIzol reagent (Invitrogen), and complementary DNA was obtained by the reverse transcription of total RNA using the MultiScribe RT kit (Applied Biosystems, Foster City, CA, USA). Then, the levels of target genes, including lncRNA MALAT1, B-cell lymphoma-2 (Bcl-2), Bax, caspase-3, EZH2, DAB2IP and Brachyury were detected using SYBR ${ }^{\circledR}$ Premix Ex Taq ${ }^{\mathrm{TM}}$ (Takara Biotechnology Co., Ltd., Dalian, China). Glyceraldehyde-3-phosphate dehydrogenase (GAPDH) served as an internal reference. Primers for these genes are listed in 
Table I. Primer sequences for specific genes.

\begin{tabular}{ll}
\hline Gene & \multicolumn{1}{c}{ Primer sequences } \\
\hline RT-qPCR & \\
MALAT1 & F: 5'-AAAGCAAGGTCTCCCCACAAG-3' \\
& R: 5'-GGTCTGTGCTAGATCAAAAGGCA-3' \\
Bcl-2 & F: 5'-CATCAGGAAGGCTAGAGTTACC-3' \\
& R: 5'-CAGACATTCGGAGACCACAC-3' \\
Bax & F: 5'-GATGCGTCCACCAAGAAG-3' \\
& R: 5'-AGTTGAAGTTGCCGTCAG-3' \\
Caspase-3 & F: 5'-TGTCATCTCGCTCTGGTACG-3' \\
& R: 5'-AAATGACCCCTTCATCACCA-3' \\
ZEH2 & F: 5'-TTGTTGGCGGAAGCGTGTAAAATC-3' \\
& R: 5'-TCCCTAGTCCCGCGCAATGAGC-3' \\
DAB2IP & F: 5'-ACACGCCATGGAGCCCGACT-3' \\
& R: 5'-GAAGCCCGTGACCCGGAACG-3' \\
Brachyury & F: 5'-AGGTGGGGAAGTTTCCTTCT-3' \\
& R: 5'-GCAAATGAGGTCCTTTTGGT-3' \\
GAPDH & F: 5'-AGCTTCGGCACATATTTCATCTG-3' \\
& R: 5'-CGTTCACTCCCATGACAAACA-3' \\
ChIP-qPCR & \\
DAB2IP & F: 5'-CCTGCTTTCTGTTTCCTTCTCCTG-3' \\
& R: 5'-TTGAACCACCTCCTCCTCCCTCTC-3' \\
& F: 5'-AGGTGGGGAAGTTTCCTTCT-3' \\
& R:-GCAAATGAGGTCCTTTTGGT-3' \\
\hline Brachyury &
\end{tabular}

RT-qPCR, real-time-quantitative polymerase chain reaction; MALAT1, metastasis-associated lung adenocarcinoma transcript 1; Bcl-2, B-cell lymphoma-2; GAPDH, glyceraldehyde-3-phosphate dehydrogenase; ChIP, chromatin immunoprecipitation; F, forward; R, reverse.

Table I. The relative quantification of these genes was carried out using the comparative threshold $(\mathrm{Ct})$ cycle method $\left(2^{-\Delta \Delta \mathrm{Ct}}\right)$.

Western blotting. RIPA buffer (Sangon Biotech, Shanghai, China) was used to extract protein from the heart tissues and cells. The measurement of protein concentration was conducted using BCA Protein Quantitative assay (Pierce, Appleton, WI, USA). Protein samples were subjected to sodium dodecyl sulfate-polyacrylamide gel electrophoresis (SDS-PAGE), and then blotted onto nitrocellulose membranes (Millipore, Billerica, MA, USA). After being blocked with 5\% nonfat milk in Tris-buffered saline Tween-20 (TBST), the membranes were incubated with rat anti-Bcl-2, Bax, caspase-3, EZH2, DAB2IP, Brachyury and GAPDH monoclonal antibodies (1:500-1,000; Santa Cruz Biotechnology, Inc., Santa Cruz, CA, USA) overnight at $4^{\circ} \mathrm{C}$, respectively. After being washed with TBST, the membranes were incubated with goat anti-rat secondary antibody (1:5,000; Santa Cruz Biotechnology, Inc.) for $2 \mathrm{~h}$ at room temperature. Ultimately, the proteins were detected with Enhanced chemiluminescence (Millipore, Bedford, MA, USA).

RNA immunoprecipitation (RIP) assay. Magna RIP RNA-Binding Protein Immunoprecipitation kit (Millipore) was used to perform RIP assay following the manufacturer's instructions. In brief, CMVECs were treated with LPS for $6 \mathrm{~h}$.
Then, the cells were collected and treated with RIPA buffer. After centrifugation, the supernatant was incubated with protein-A/G-Sepharose beads, anti-EZH2 monoclonal antibody and $\mathrm{IgG}$ overnight at $4^{\circ} \mathrm{C}$. Subsequently, co-precipitated RNA was extracted and then detected by RT-qPCR.

Chromatin immunoprecipitation (ChIP) assay. ChIP assay was carried out using QuikChIP kit (Imgenex, San Diego, CA, USA). In brief, CMVECs were transfected with two siRNAs targeting MALAT1 (si-MALAT1-1 and si-MALAT1-2; Dharmacon, Mickleton, NJ, USA) using Lipofectamine 2000 for $48 \mathrm{~h}$. Then, protein-chromatin cross-linking of cells was induced using $1 \%$ formaldehyde for $10 \mathrm{~min}$ at $37^{\circ} \mathrm{C}$ and quenched by glycine. Next, the cells were lysed in lysis buffer and chromatin DNA from cell lysates was sonicated into fragments. Next, chromatin DNA fragments were subjected to immunoprecipitation with EZH2 or methyltransferase catalyzing histone $\mathrm{H} 3$ lysine 27 trimethylation (H3K27me3) antibody. After reverse cross-linking, DNA fragments were purified and then detected by RT-qPCR.

Statistical analysis. Statistical analyses were carried out using SPSS 19.0 (IBM Corp., Armonk, NY, USA). The results of all experiments are expressed as the mean \pm SD and analyzed by t-test. A statistically significant difference was defined as a p-value $<0.05$.

\section{Results}

Ulinastatin reduces LPS-induced CMVEC hyperpermeability. The results of TER showed that compared with the untreated cells, cell permeability was significantly increased $(\mathrm{p}<0.05)$ in the LPS-induced cells in a dose- and time-dependent manner (Fig. 1A and B). Notably, cell permeability was elevated when the dose of LPS exceeded $50 \mathrm{ng} / \mathrm{ml}$ and the treatment time was $>6 \mathrm{~h}$ (Fig. $1 \mathrm{~A}$ and B). Thus, the optimum inducement time and concentration was determined as $6 \mathrm{~h}$ and $50 \mathrm{ng} / \mathrm{ml}$. In addition, the presence of ulinastatin markedly inhibited $(\mathrm{p}<0.05)$ cell permeability in LPS-induced cells, and 5,000 U/1 ulinastatin led to the more obvious improvement (Fig. 1C).

Ulinastatin reduces LPS-induced CMVEC apoptosis. Next, we analyzed the effect of ulinastatin on CMVEC apoptosis. The results revealed that compared with the untreated cells, the percentage of apoptotic cells was markedly increased in the LPS-induced cells $(\mathrm{p}<0.01)$, while it was obviously decreased after treatment with ulinastatin $(\mathrm{p}<0.05)$ (Fig. 2A). Meanwhile, the expression levels of cell apoptosis-related proteins, including Bcl-2, caspase-3 and Bax, were detected by RT-qPCR and western blotting, respectively. The results showed that LPS-induced cells showed a lower Bcl-2 level as well as higher Bax and caspase-3 levels than the untreated cells $(p<0.05)$ (Fig. 2B). After treatment with ulinastatin, the mRNA and protein levels of Bcl-2 were significantly increased, and the levels of caspase- 3 and Bax were markedly decreased in the LPS-induced cells ( $\mathrm{p}<0.05)$ (Fig. 2B). Also, compared with the untreated cells, LPS induced a higher ROS level $(\mathrm{p}<0.01)$ (Fig. 2C), while ulinastatin markedly inhibited the increase in ROS level induced by LPS ( $\mathrm{p}<0.05)$ (Fig. 2C). 


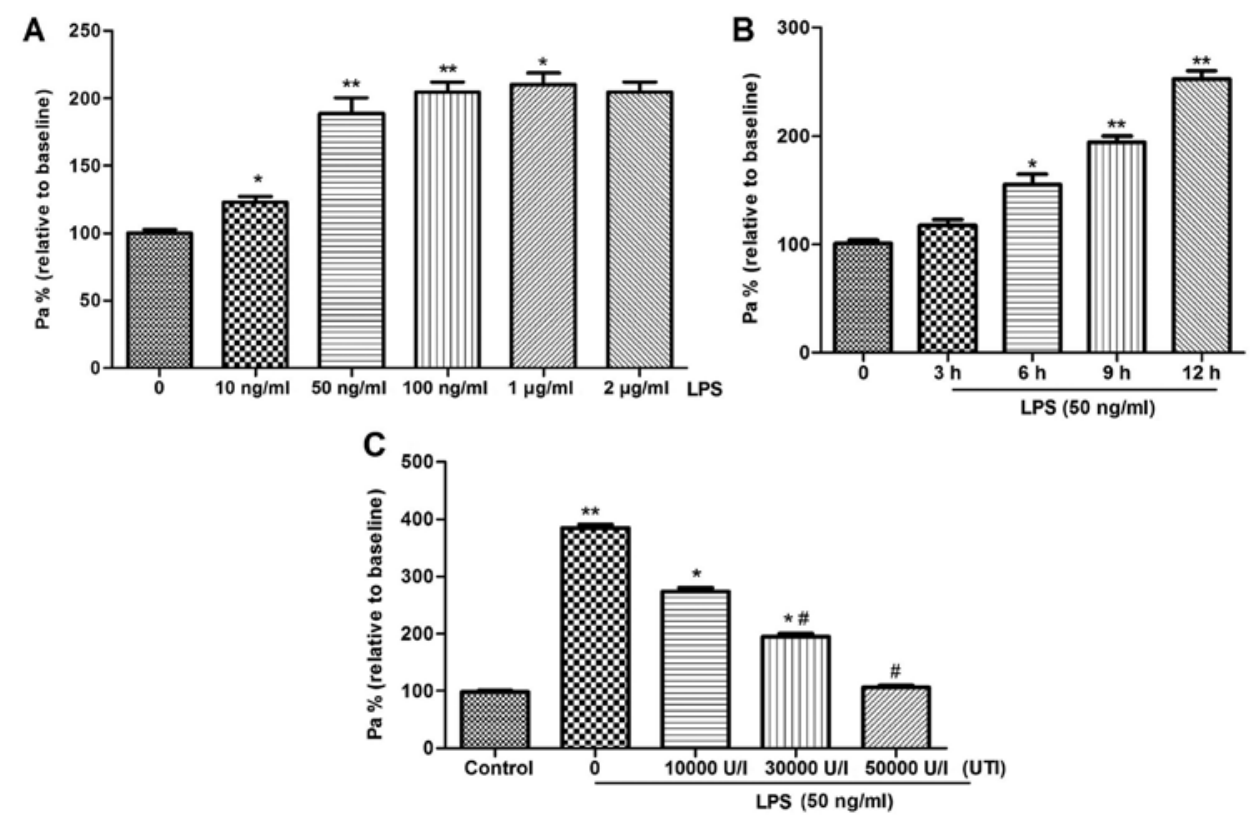

Figure 1. Ulinastatin inhibits cell permeability in lipopolysaccharide (LPS)-induced cardiac microvascular endothelial cells (CMVECs). (A) Cell permeability in CMVECs treated with different doses of LPS for $6 \mathrm{~h}$ by transendothelial electrical resistance (TER). (B) Cell permeability of the CMVECs treated with $50 \mathrm{ng} / \mathrm{ml}$ LPS for different times as determined by the TER. (C) Cell permeability of the CMVECs treated with $50 \mathrm{ng} / \mathrm{ml}$ LPS and/or different doses of ulinastatin (UTI) for $6 \mathrm{~h}$ as determined by TER. ${ }^{*} \mathrm{p}<0.05$ and ${ }^{* *} \mathrm{p}<0.01$ compared with the control group; ${ }^{*} \mathrm{p}<0.05$ compared with the LPS group.
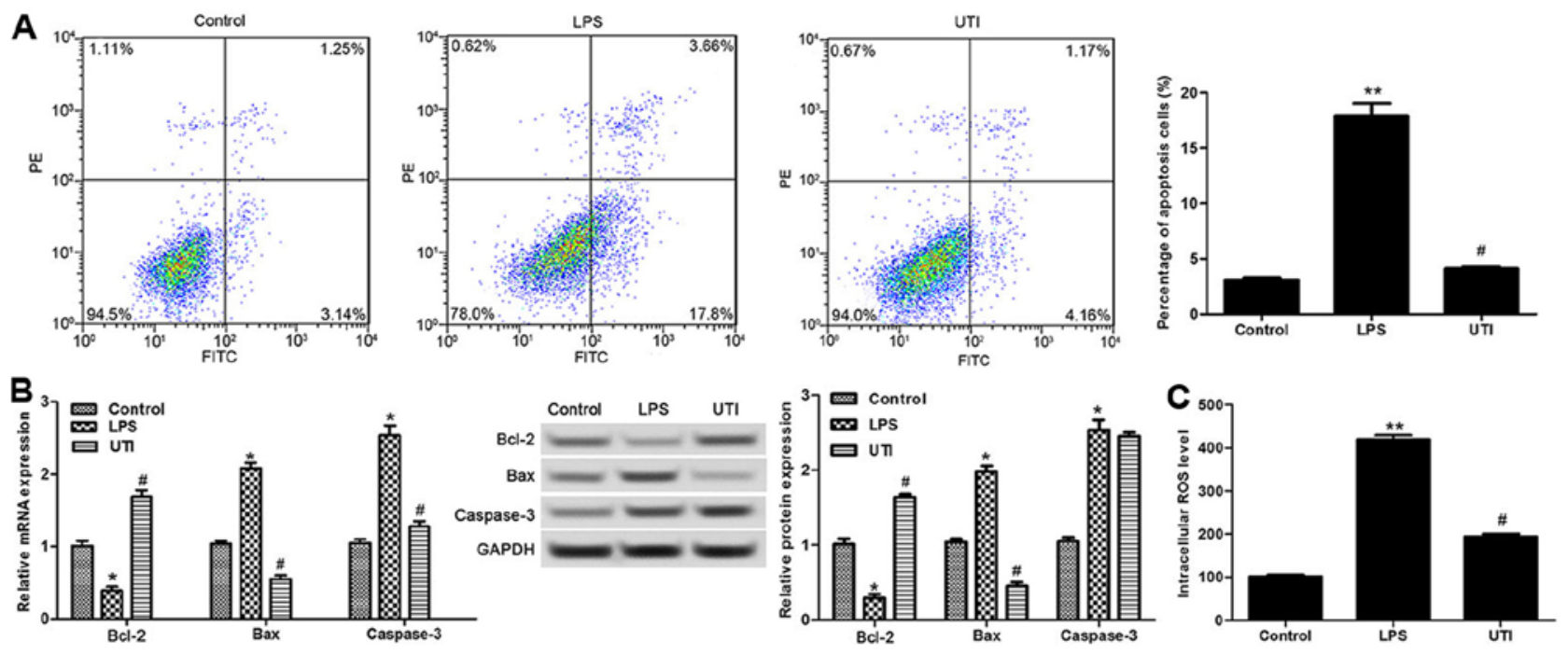

Figure 2. Ulinastatin inhibits cell apoptosis in lipopolysaccharide (LPS)-induced cardiac microvascular endothelial cells (CMVECs). (A) The percentage of apoptotic cells in the LPS-induced CMVECs with or without treatment with ulinastatin (UTI) using Annexin V-FITC apoptosis detection kit. (B) The expression levels of cell apoptosis-related proteins, including Bcl-2, caspase-3 and Bax, in LPS-induced CMVECs with or without treatment with UTI as detected by RT-qPCR and western blotting. (C) Reactive oxygen species (ROS) level in LPS-induced CMVECs with or without treatment with UTI. ${ }^{*}<<0.05$ and ${ }^{* *}$ p $<0.01$ compared with the control group; ${ }^{*} \mathrm{p}<0.05$ compared with the LPS group.

Ulinastatin inhibits the expression of IncRNA MALAT1 and EZH2 in LPS-induced CMVECs. To investigate the mechanism of ulinastatin in LPS-induced CMVECs, the expression levels of lncRNA MALAT1 and EZH2 were detected. The results revealed that the levels of IncRNA MALAT1 and EZH2 were significantly upregulated in the hearts of rat from the sepsis group in comparison with these levels in the sham group $(\mathrm{p}<0.01)$ (Fig. 3A). Similarly, in vitro experiments showed that LPS markedly elevated the expression levels of 1ncRNA MALAT1 and EZH2 compared with the levels in the untreated cells $(\mathrm{p}<0.01)$ (Fig. 3B), while ulinastatin inhibited the upregulation of lncRNA MALAT1 and EZH2 $(\mathrm{p}<0.05)$ (Fig. 3B).

lncRNA MALAT1 interacts with EZH2 in CMVECs. RTP assay was performed to evaluate the relationship between IncRNA MALAT1 and EZH2. The results revealed that lncRNA MALAT1 could bind to EZH2 (Fig. 4A), indicating the interaction of lncRNA MALAT1 and EZH2. Then, the lncRNA MALAT1 level was successfully inhibited by the transfection of si-MALAT1-1 and si-MALAT1-2 (p<0.05) (Fig. 4B). Silencing of lncRNA MALAT1 markedly inhibited the expression of 


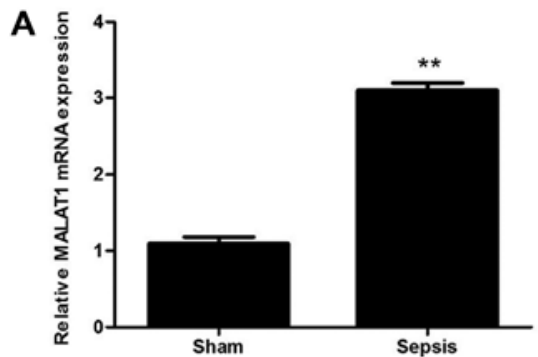

B
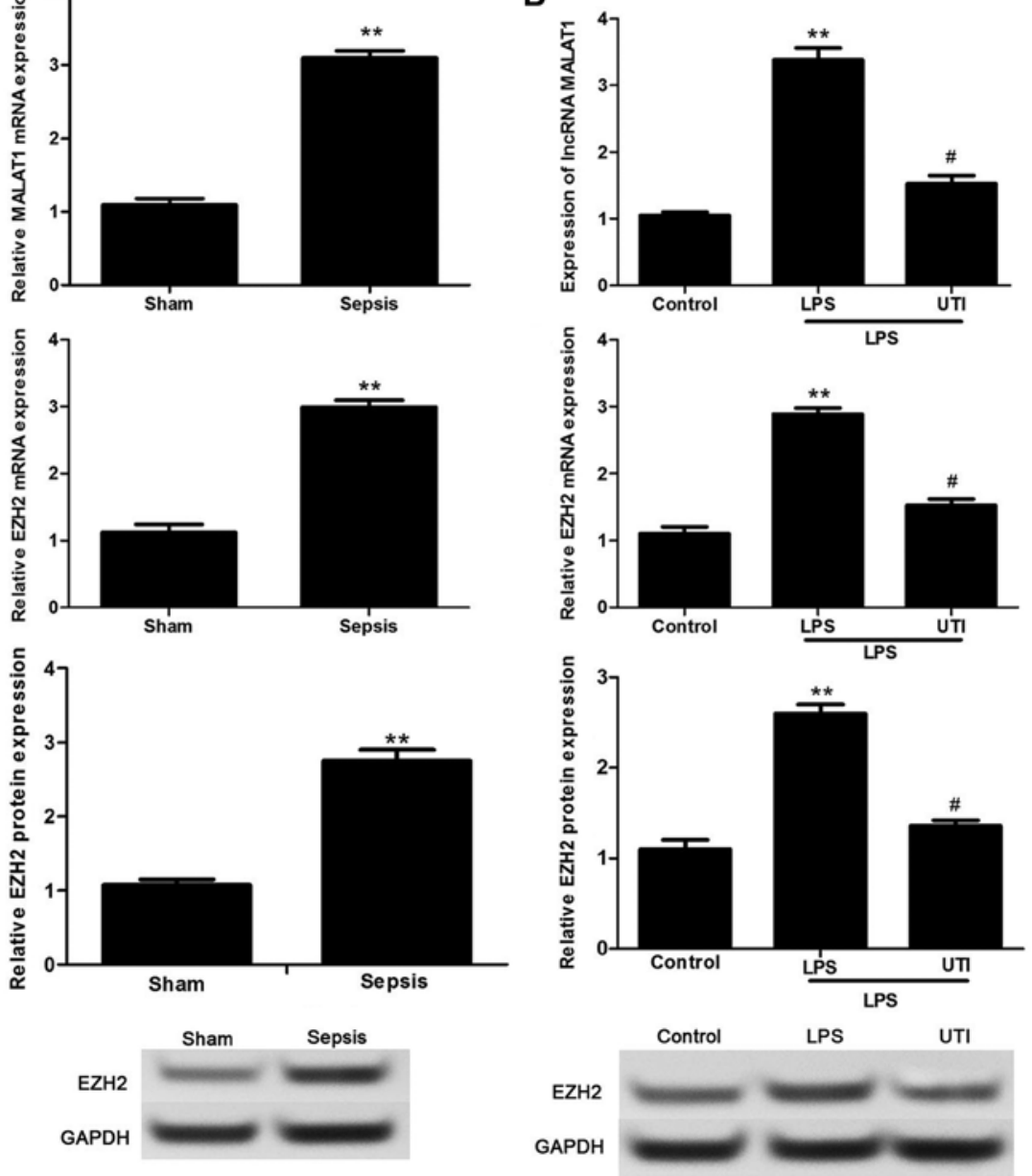

Figure 3. Ulinastatin inhibits the levels of long non-coding RNA (lncRNA) metastasis-associated lung adenocarcinoma transcript 1 (MALAT1) and EZH2 in lipopolysaccharide (LPS)-induced cardiac microvascular endothelial cells (CMVECs). (A) The levels of lncRNA MALAT1 and EZH2 in the hearts of sepsis and sham rats. (B) The levels of lncRNA MALAT1 and EZH2 in the LPS-induced CMVECs with or without ulinastatin (UTI) treatment. "p $<0.05$ and ${ }^{* *} \mathrm{p}<0.01$ compared with the control group; ${ }^{\#} \mathrm{p}<0.05$ compared with the LPS group.
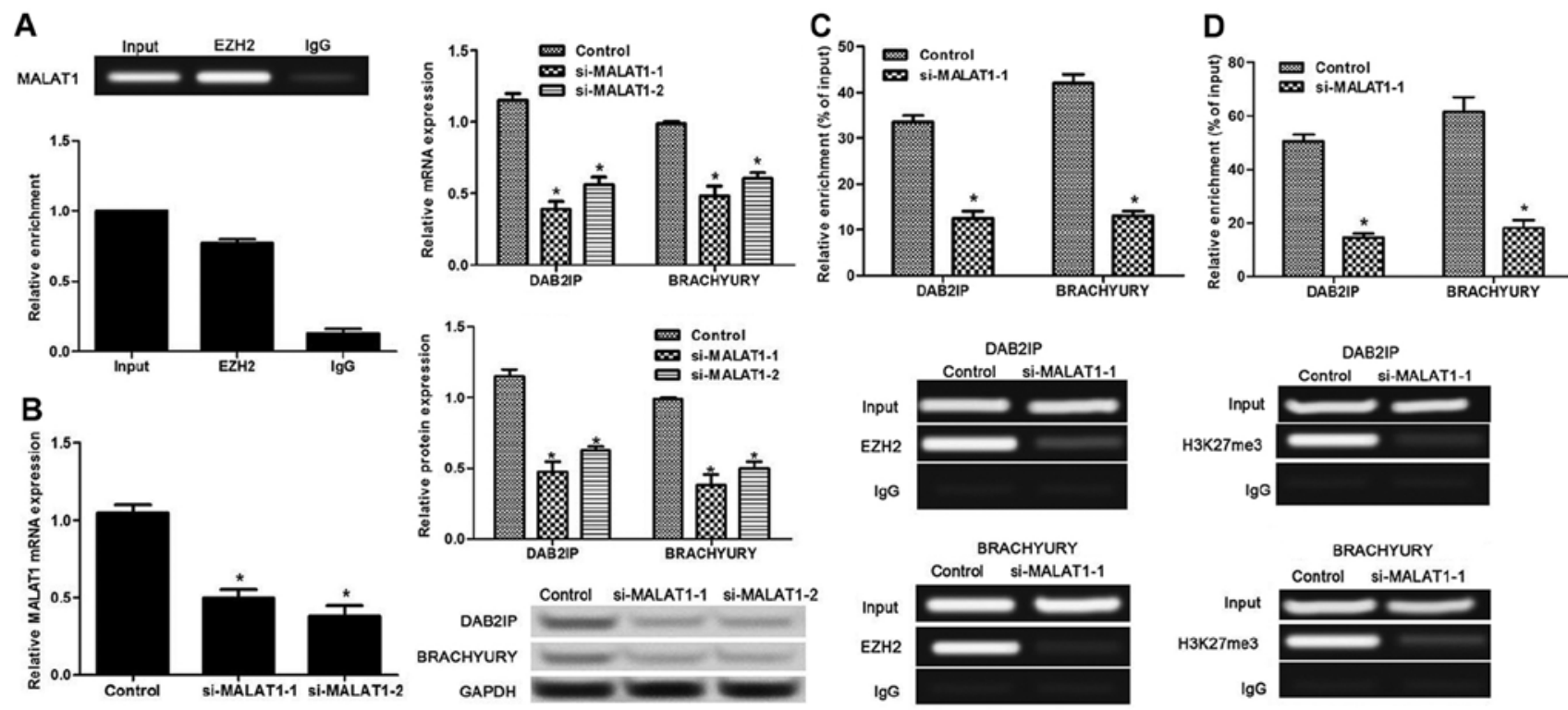

Figure 4. Metastasis-associated lung adenocarcinoma transcript 1 (MALAT1) interacts with EZH2 in cardiac microvascular endothelial cells (CMVECs) (A) RNA immunoprecipitation assay confirmed that long non-coding RNA (lncRNA) MALAT1 could bind to EZH2. (B) The levels of MALAT1 as well as EZH2 target genes DAB2IP and Brachyury in CMVECs transfected with si-MALAT1-1 and si-MALAT1-2. (C) The levels of DAB2IP and Brachyury in CMVECs when MALAT1-knockdown cells were precipitated with EZH2 by chromatin immunoprecipitation (ChIP) assay. (D) The levels of DAB2IP and Brachyury in CMVECs when MALAT1-knockdown cells were precipitated with histone H3 lysine 27 trimethylation (H3K27me3) by ChIP assay. "p<0.05 compared with the control group. 
A

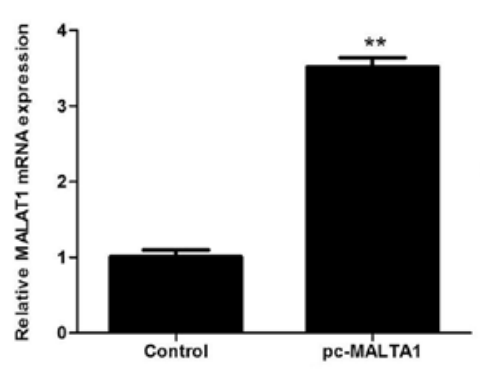

C

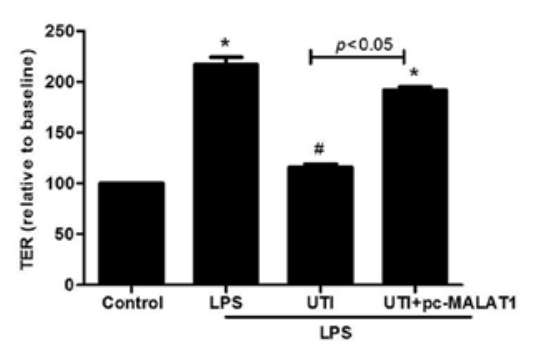

B
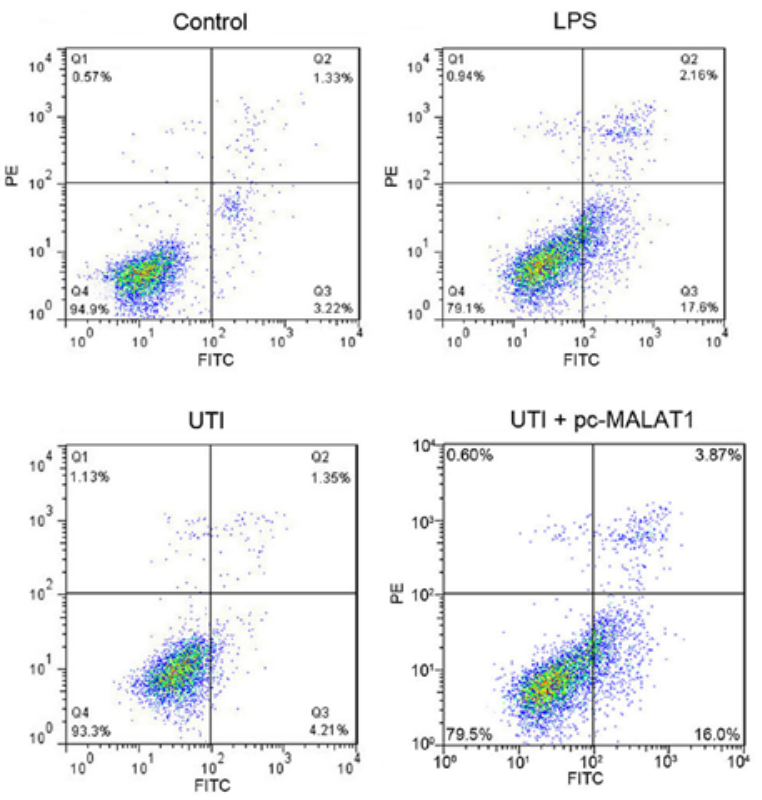

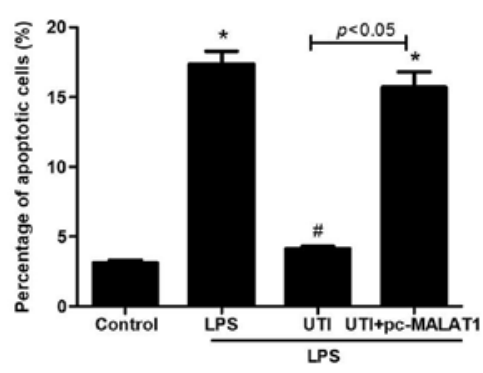

Figure 5. Overexpression of ulinastatin inhibits cell permeability and apoptosis in lipopolysaccharide (LPS)-induced cardiac microvascular endothelial cells (CMVECs). (A) The level of metastasis-associated lung adenocarcinoma transcript 1 (MALAT1) in CMVECs transfected with pc-MALAT1 as determined by RT-qPCR. (B) The percentage of apoptotic cells in CMVECs transfected with pc-MALAT1 after treatment with LPS and/or ulinastatin (UTI) using Annexin V-FITC apoptosis detection kit. (C) Cell permeability in CMVECs transfected with pc-MALAT1 after treatment with LPS and/or UTI as determined by transendothelial electrical resistance. ${ }^{*} \mathrm{p}<0.05$ and ${ }^{* *} \mathrm{p}<0.01$ compared with the control group; ${ }^{*} \mathrm{p}<0.05$ compared with the LPS group.

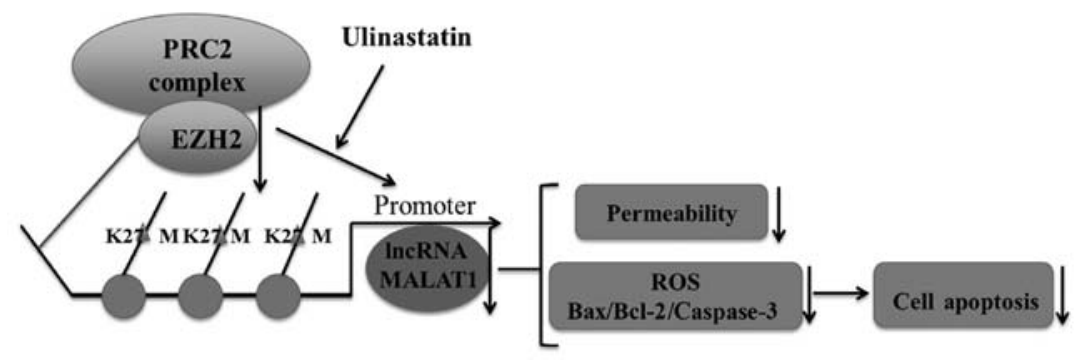

Figure 6. Diagram of the mechanism of ulinastatin in lipopolysaccharide (LPS)-induced cardiac microvascular endothelial cells (CMVECs).

EZH2 target genes, DAB2IP and Brachyury (p<0.05) (Fig. 4B) compared with that in the untreated cells. Furthermore, the effect of IncRNA MALAT1 on EZH2 was evaluated by ChIP assay. The results revealed that when lncRNA MALAT1-knockdown cells were precipitated with EZH2 or H3K27me3 (the composition of EZH2) antibody, the expression levels of DAB2IP and Brachyury were both inhibited compared with levels in the untreated cells $(\mathrm{p}<0.05)$ (Fig. 4C and D).

Ulinastatin protects against LPS-induced CMVEC dysfunction via downregulation of IncRNA MALAT1 and EZH2. The above results showed that ulinastatin induced the downregulation of IncRNA MALAT1 in the LPS-induced cells. Thus, we investigated the effect of overexpression of lncRNA MALAT1 on CMVEC permeability and apoptosis following treatment with ulinastatin and LPS. Compared with the untreated cells, the lncRNA MALAT1 level was significantly increased $(\mathrm{p}<0.01)$ (Fig. 5A) in cells transfected with pc-MALAT1. Then, the results revealed that upon treatment with ulinastatin, upregulation of lncRNA MALAT1 markedly increased CMEC permeability (Fig. 5B) and apoptosis (Fig. 5C) in the LPS-induced cells (all p<0.05). 


\section{Discussion}

Cardiac microvascular dysfunction in sepsis has been demonstrated (5) and the protective role of ulinastatin against sepsis has also been reported (22). Nevertheless, the effects of ulinastatin on CMVEC dysfunction in sepsis are still unclear. The present study revealed that compared with LPS-induced CMVECs, the treatment of ulinastatin significantly reduced CMVEC permeability and apoptosis. In addition, we found upregulated lncRNA MALAT1 and EZH2 in the hearts of sepsis rats and LPS-induced CMVECs, while ulinastatin had an inhibitory effect on the upregulation of lncRNA MALAT1 and $\mathrm{EZH} 2$.

Several studies have confirmed the LPS-induced sepsis cell or animal model $(23,24)$. CMVEC dysfunction has been reported to be associated with the occurrence of sepsis $(5,25)$. This study found that LPS treatment promoted CMVEC dysfunction, such as hyperpermeability and apoptosis. Consistently, Janicek et al (26) demonstrated the hyperpermeability and apoptosis in endothelial cells induced by LPS. In addition, we found that ulinastatin reduced LPS-induced CMVEC hyperpermeability and apoptosis. Previous studies have shown that ulinastatin had inhibitory effects on apoptosis, oxidative stress and inflammation $(9,27)$. The protective effect of ulinastatin against vascular endothelial injury was also reported in patients who underwent heart operation (28). Moreover, Li et al (29) reported that ulinastatin inhibited cell hyperpermeability and apoptosis in oxidant-induced human umbilical endothelial cells. Lin et al (30) also revealed that ulinastatin attenuated the hyperpermeability of rat lung microvascular endothelial cells when treated by shock serum. All these results collaborate our results. The present study further revealed that ulinastatin inhibited the levels of ROS, caspase-3 and Bax as well as increased the level of Bcl-2 in LPS-induced CMVECs. It was well known that ROS induce mitochondrial oxidative stress and then promote the release of cytochrome $c$ (31). Oxidative stress plays a vital regulatory role in mitochondria dependent apoptotic signaling (32). Mitochondria-mediated apoptosis could be regulated by anti-apoptotic factor Bcl-2, pro-apoptotic factor Bax (26), as well as caspase- 3 activation, which increase membrane permeability $(32,33)$. These results suggest that ulinastatin inhibits LPS-induced cell hyperpermeability and apoptosis through a mitochondrial-dependent pathway.

The present study further investigated the role of lncRNA MALAT1 in CMVEC dysfunction in sepsis. The results revealed the upregulation of 1ncRNA MALAT1 and EZH2 in vitro and in vivo sepsis model, while ulinastatin inhibited the expression of 1ncRNA MALAT1 and EZH2. Previous studies have shown overexpression of MALAT1 in cancers, including non-small cell lung (34), bladder (35), colorectal (36) and prostate cancer (37). Recently, MALAT1 has been reported to regulate the production of inflammatory cytokines in cancers (38). Polycomb protein EZH2 was also reported to be overexpressed in cancers (39). EZH2 was considered as an oncogenic gene that interacts with EED and SUZ12 by forming the polycomb repressive complex-2 (PRC2) (40). H3K27me3 is a key functional component of EZH2 (40). This study revealed that IncRNA MALAT1 could bind to EZH2, and MALAT1 silencing inhibited the expression of EZH2 target genes $D A B 2 I P$ and Brachyury in cells precipitated with $\mathrm{EZH} 2$ or $\mathrm{H} 3 \mathrm{~K} 27 \mathrm{me} 3$. These results indicated the interaction of MALAT1 and EZH2 in CMVECs, which was consistent with the study of Wang et al (41). Increasing evidence has shown that both MALAT1 and EZH2 are associated with cell proliferation, apoptosis and metastasis in cancer cells $(42,43)$. All these results indicate that ulinastatin may inhibit LPS-induced CMVEC dysfunction by inhibiting lncRNA MALAT1 and EZH2. The diagram illustrating the mechanism of ulinastatin in LPS-induced CMVECs is shown in Fig. 6.

Ulinastatin protects against LPS-induced CMVEC hyperpermeability and apoptosis via downregulation of lncRNA MALAT1 and EZH2 in sepsis.

\section{Acknowledgements}

This study was supported by the Graduate Innovation Fund of Xinjiang Medical University (grant no. CXCY002).

\section{References}

1. Sprung CL and Reinhart K: Definitions for sepsis and septic shock. JAMA 316: 456-457, 2016.

2. Brun-Buisson C, Meshaka P, Pinton P and Vallet B; EPISEPSIS Study Group: EPISEPSIS: A reappraisal of the epidemiology and outcome of severe sepsis in French intensive care units. Intensive Care Med 30: 580-588, 2004.

3. Gaieski DF,Edwards JM, Kallan MJ and Carr BG: Benchmarking the incidence and mortality of severe sepsis in the United States. Crit Care Med 41: 1167-1174, 2013.

4. Zaky A,Deem S, Bendjelid K and Treggiari MM: Characterization of cardiac dysfunction in sepsis: An ongoing challenge. Shock 41: $12-24,2014$.

5. Lush CW and Kvietys PR: Microvascular dysfunction in sepsis. Microcirculation 7: 83-101, 2000.

6. Scarabelli T, Stephanou A, Rayment N, Pasini E, Comini L, Curello S, Ferrari R, Knight R and Latchman D: Apoptosis of endothelial cells precedes myocyte cell apoptosis in ischemia/reperfusion injury. Circulation 104: 253-256, 2001.

7. Salmon AH and Satchell SC: Endothelial glycocalyx dysfunction in disease: Albuminuria and increased microvascular permeability. J Pathol 226: 562-574, 2012.

8. Liu Y, Lian K, Zhang L, Wang R, Yi F, Gao C, Xin C, Zhu D, Li Y, Yan W, et al: TXNIP mediates NLRP3 inflammasome activation in cardiac microvascular endothelial cells as a novel mechanism in myocardial ischemia/reperfusion injury. Basic Res Cardiol 109: 415, 2014.

9. Xu CE, Zhang MY, Zou CW and Guo L: Evaluation of the pharmacological function of ulinastatin in experimental animals. Molecules 17: 9070-9080, 2012.

10. Wang N, Liu X, Zheng X, Cao H, Wei G, Zhu Y, Fan S, Zhou H and Zheng J: Ulinastatin is a novel candidate drug for sepsis and secondary acute lung injury, evidence from an optimized CLP rat model. Int Immunopharmacol 17: 799-807, 2013.

11. Cao YZ, Tu YY, Chen X, Wang BL, Zhong YX and Liu MH: Protective effect of Ulinastatin against murine models of sepsis: Inhibition of TNF- $\alpha$ and IL- 6 and augmentation of IL-10 and IL-13. Exp Toxicol Pathol 64: 543-547, 2012.

12. Huang N, Wang F, Wang Y, Hou J, Li J and Deng X: Ulinastatin improves survival of septic mice by suppressing inflammatory response and lymphocyte apoptosis. J Surg Res 182: 296-302, 2013.

13. Karnad DR, Bhadade R, Verma PK, Moulick ND, Daga MK, Chafekar ND and Iyer S: Intravenous administration of ulinastatin (human urinary trypsin inhibitor) in severe sepsis: A multicenter randomized controlled study. Intensive Care Med 40: 830-838, 2014.

14. Bashir F, Rather MA, Saleem B and Hamid A: a prospective, randomized study using ulinastatin for the treatment of patients with severe sepsis. JEMDS 3: 12241-12246, 2014.

15. Shi X, Sun M, Liu H, Yao Y and Song Y: Long non-coding RNAs: A new frontier in the study of human diseases. Cancer Lett 339: 159-166, 2013.

16. Johnson R: Long non-coding RNAs in Huntington's disease neurodegeneration. Neurobiol Dis 46: 245-254, 2012. 
17. Congrains A, Kamide K, Oguro R, Yasuda O, Miyata K, Yamamoto E, Kawai T, Kusunoki H, Yamamoto H, Takeya Y, et al: Genetic variants at the 9p21 locus contribute to atherosclerosis through modulation of ANRIL and CDKN2A/B. Atherosclerosis 220: 449-455, 2012.

18. Spizzo R, Almeida MI, Colombatti A, Calin GA and Martin L: Long non-coding RNAs and cancer: A new frontier of translational research? Oncogene 31: 4577-4587, 2012.

19. Guo F, Li Y, Liu Y, Wang J, Li Y and Li G: Inhibition of metastasis-associated lung adenocarcinoma transcript 1 in CaSki human cervical cancer cells suppresses cell proliferation and invasion. Acta Biochim Biophys Sin (Shanghai) 42: 224-229, 2010.

20. Brooks HF, Moss RF, Davies NA, Jalan R and Davies DC: Caecal ligation and puncture induced sepsis in the rat results in increased brain water content and perimicrovessel oedema. Metab Brain Dis 29: 837-843, 2014

21. Xia JB, Liu GH, Chen ZY, Mao CZ, Zhou DC, Wu HY, Park KS, Zhao H, Kim SK, Cai DQ, et al: Hypoxia/ischemia promotes CXCL10 expression in cardiac microvascular endothelial cells by NFKB activation. Cytokine 81: 63-70, 2016.

22. Wang FY, Fang B, Qiang XH, Yu TO, Zhong JR, Cao J and Zhou LX: The efficacy and immunomodulatory effects of ulinastatin and thymosin $\alpha 1$ for sepsis: A systematic review and meta-analysis. Biomed Res Int 2016: 9508493, 2016.

23. Tsoyi K, Lee TY,Lee YS, Kim HJ, Seo HG, Lee JH and Chang KC: Heme-oxygenase-1 induction and carbon monoxide-releasing molecule inhibit lipopolysaccharide (LPS)-induced high-mobility group box 1 release in vitro and improve survival of mice in LPS-and cecal ligation and puncture-induced sepsis model in vivo. Mol Pharmacol 76: 173-182, 2009.

24. Djoumerska-Alexieva I, Pashova S, Vassilev T and Pashov A: The protective effect of modified intravenous immunoglobulin in LPS sepsis model is associated with an increased IRA B cells response. Autoimmun Rev 12: 653-656, 2013.

25. Lee WL and Slutsky AS: Sepsis and endothelial permeability. N Engl J Med 363: 689-691, 2010.

26. Janicek A, Tharakan B, Sawant DA, Hunter FA and Childs EW: Lipopolysaccharide-induced endothelial cell hyperpermeability: Role of mitochondrial apoptotic signaling pathway. J Surg Res 172: 330, 2012.

27. Hu CL, Xia JM, Cai J, Li X, Liao XX, Li H, Zhan H, Dai G and Jing XL: Ulinastatin attenuates oxidation, inflammation and neural apoptosis in the cerebral cortex of adult rats with ventricular fibrillation after cardiopulmonary resuscitation. Clinics (Sao Paulo) 68: 1231-1238, 2013.

28. Miura M, Sugiura T, Aimi Y, Yasuda K, Ito S, Baba E and Katsuya H: Effects of ulinastatin on PMNL and vascular endothelial injury in patients undergoing open heart surgery with CPB. Masui 47: 29-35, 1998 (In Japanese).

29. Li G, Li T, Li Y, Cai S, Zhang Z, Zeng Z, Wang X, Gao Y, Li Y and Chen Z: Ulinastatin inhibits oxidant-induced endothelial hyperpermeability and apoptotic signaling. Int J Clin Exp Pathol 7: 7342-7350, 2014.

30. Lin B, Liu Y, Li T, Zeng K, Cai S, Zeng Z, Lin C, Chen Z and Gao Y: Ulinastatin mediates protection against vascular hyperpermeability following hemorrhagic shock. Int J Clin Exp Pathol 8: 7685-7693, 2015.
31. Atlante A, Calissano P, Bobba A, Azzariti A, Marra E and Passarella S: Cytochrome $c$ is released from mitochondria in a reactive oxygen species (ROS)-dependent fashion and can operate as a ROS scavenger and as a respiratory substrate in cerebellar neurons undergoing excitotoxic death. J Biol Chem 275: 37159-37166, 2000

32. Sinha K, Das J, Pal PB and Sil PC: Oxidative stress: The mitochondria-dependent and mitochondria-independent pathways of apoptosis. Arch Toxicol 87: 1157-1180, 2013.

33. Childs EW, Tharakan B, Hunter FA, Tinsley JH and Cao X: Apoptotic signaling induces hyperpermeability following hemorrhagic shock. Am J Physiol Heart Circ Physiol 292: H3179-H3189, 2007.

34. Ji P, Diederichs S, Wang W, Böing S, Metzger R, Schneider PM, Tidow N, Brandt B, Buerger H, Bulk E, et al: MALAT-1, a novel noncoding RNA, and thymosin beta4 predict metastasis and survival in early-stage non-small cell lung cancer. Oncogene 22: 8031-8041, 2003.

35. Ying L, Chen Q, Wang Y, Zhou Z, Huang Y and Qiu F: Upregulated MALAT-1 contributes to bladder cancer cell migration by inducing epithelial-to-mesenchymal transition. Mol Biosyst 8: 2289-2294, 2012.

36. Xu C, Yang M, Tian J, Wang X and Li Z: MALAT-1: a long non-coding RNA and its important 3 ' end functional motif in colorectal cancer metastasis. Int J Oncol 39: 169-175, 2011.

37. Ren S, Liu Y, Xu W, Sun Y, Lu J, Wang F, Wei M, Shen J, Hou J, Gao X, et al: Long noncoding RNA MALAT-1 is a new potential therapeutic target for castration resistant prostate cancer. J Urol 190: 2278-2287, 2013.

38. Gutschner T, Hämmerle M and Diederichs S: MALAT1 - a paradigm for long noncoding RNA function in cancer. J Mol Med (Berl) 91: 791-801, 2013.

39. Bachmann IM, Halvorsen OJ, Collett K, Stefansson IM, Straume O, Haukaas SA, Salvesen HB, Otte AP and Akslen LA: EZH2 expression is associated with high proliferation rate and aggressive tumor subgroups in cutaneous melanoma and cancers of the endometrium, prostate, and breast. J Clin Oncol 24: 268-273, 2006.

40. Cao Q, Yu J, Dhanasekaran SM, Kim JH, Mani RS, Tomlins SA, Mehra R, Laxman B, Cao X, Yu J, et al: Repression of E-cadherin by the polycomb group protein EZH2 in cancer. Oncogene 27: 7274-7284, 2008

41. Wang D, Ding L, Wang L, Zhao Y, Sun Z, Karnes RJ, Zhang J and Huang H: LncRNA MALAT1 enhances oncogenic activities of EZH2 in castration-resistant prostate cancer. Oncotarget 6: 41045-41055, 2015.

42. Xie L, Zhang Z, Tan Z, He R, Zeng X, Xie Y, Li S, Tang G, Tang $\mathrm{H}$ and He X: MicroRNA-124 inhibits proliferation and induces apoptosis by directly repressing EZH2 in gastric cancer. Mol Cell Biochem 392: 153-159, 2014

43. Jiao F, Hu H, Yuan C, Wang L, Jiang W, Jin Z, Guo Z and Wang L: Elevated expression level of long noncoding RNA MALAT-1 facilitates cell growth, migration and invasion in pancreatic cancer. Oncol Rep 32: 2485-2492, 2014 